\title{
CONVERGENCE ANALYSIS OF AN ITERATIVE CORRELATION-BASED CONTROLLER TUNING METHOD
}

\author{
A. Karimi, L. Miskovic and D. Bonvin \\ Institut d'Automatique, EPFL, CH-1015 Lausanne, Switzerland. \\ Fax:0041(21)6932574,e-mail: alireza.karimi@epfl.ch
}

\begin{abstract}
A new iterative method using closed-loop data for controller tuning based on the correlation approach is proposed. The main idea is to make the output error between the closed-loop system and a reference model uncorrelated with the reference signal. The controller parameters are calculated as the solution to a correlation equation involving instrumental variables. Convergence and consistency of the controller parameters for two choices of instrumental variables are analyzed. It is shown that the controller parameters converge to their true values independent of the noise characteristics and modeling error. Simulation results confirm the effectiveness of the proposed approach.
\end{abstract}

Keywords: Controller tuning, instrumental variables, convergence analysis.

\section{INTRODUCTION}

Control problems are generally expressed as the minimization of an error signal. In many servo control problems, the error signal may be defined as the difference between the output of the closedloop system and the output of a reference model that represents the desired response of the closedloop system to a reference signal. This problem is called model following and can be solved using pole-placement design provided that the plant model is perfectly known. For the case of unknown plant models or models with time-variant parameters, Self-Tuning Regulation (STR) or ModelReference Adaptive Control (MRAC) can be employed (Åström and Wittenmark, 1989). In these approaches, optimization methods are used to find the controller parameters driving the error signal to zero. The approaches can be extended to the case where a general quadratic criterion is minimized. The gradient of the criterion is calculated using an on-line estimated model of the plant (Trulsson and Ljung, 1985) or using closed-loop data as in the Iterative Feedback Tuning (IFT) approach (Hjalmarsson et al., 1994). However, a characteristic feature of these approaches is that, in the presence of noise, the controller parameters do not necessarily converge to their correct values (the values computed from the true plant model). As an extreme case, if the excitation signal is kept constant, a minimum-variance controller is obtained, which is known to lack robustness.

In this paper, a new approach to model-following problem based on correlation technique is introduced and its convergence is studied. The main idea is to modify the control objective so that, instead of minimizing a norm of the error signal, one tries to make the closed-loop output error (the difference between the output of the closedloop system and the reference model) uncorrelated with the excitation signal. This way, the achieved closed-loop system will capture the dynamics of the reference model (i.e., the desired dynamics) such that there remains no information about the excitation signal in the closed-loop output error. Thus, this error will mainly contain the contribution of noise that is uncorrelated with the excitation signal. 
In contrast to MRAC, STR and IFT, the effect of noise on the closed-loop output is not minimized in this approach. In fact, the designed closed-loop model (reference model) is approximated by the achieved one, independently of the noise characteristics. As a result, the robustness properties of the designed closed-loop system will be preserved, and the performance with respect to noise attenuation is not changed.

The paper is organized as follows. In Section 2, the notations and the basics of the correlation approach and the choice of instrumental variables are presented. The convergence and the consistency of the algorithm for different choices of the instruments are studied in Section 3. Simulation results are given in Section 4. Finally, Section 5 concludes the paper.

\section{CORRELATION APPROACH}

A SISO linear time-invariant discrete-time system is considered as the plant model. Let the output $y(t)$ of the system be described as:

$$
y(t)=G\left(q^{-1}\right) u(t)+v(t)
$$

where $u(t)$ is the plant input, $v(t)$ represents a zero-mean noise and the transfer operator $G\left(q^{-1}\right)$ is defined as:

$$
G\left(q^{-1}\right)=\frac{B\left(q^{-1}\right)}{A\left(q^{-1}\right)}
$$

This system is controlled by the control law:

$$
u(t)=\frac{S\left(q^{-1}\right)}{R\left(q^{-1}\right)}[r(t)-y(t)]
$$

where

$$
\begin{aligned}
& R\left(q^{-1}\right)=1+r_{1} q^{-1}+\cdots+r_{n_{R}} q^{-n_{R}} \\
& S\left(q^{-1}\right)=s_{0}+s_{1} q^{-1}+\cdots+s_{n_{S}} q^{-n_{S}}
\end{aligned}
$$

and $r(t)$ is the reference or excitation signal. The controller output can be presented in regression form as:

$$
u(t)=\phi^{T}(\rho, t) \rho
$$

with the regressor vector $\phi(\rho, t)$ and the vector of controller parameters $\rho$, both of dimension $n_{\rho}$, defined as:

$$
\begin{aligned}
\phi^{T}(\rho, t)= & {\left[-u(t-1) \cdots-u\left(t-n_{R}\right),\right.} \\
& \left.e(t) \cdots e\left(t-n_{S}\right)\right] \\
\rho^{T}= & {\left[r_{1} \cdots r_{n_{R}}, s_{0} \cdots s_{n_{S}}\right] }
\end{aligned}
$$

and $e(t)=r(t)-y(t)$.

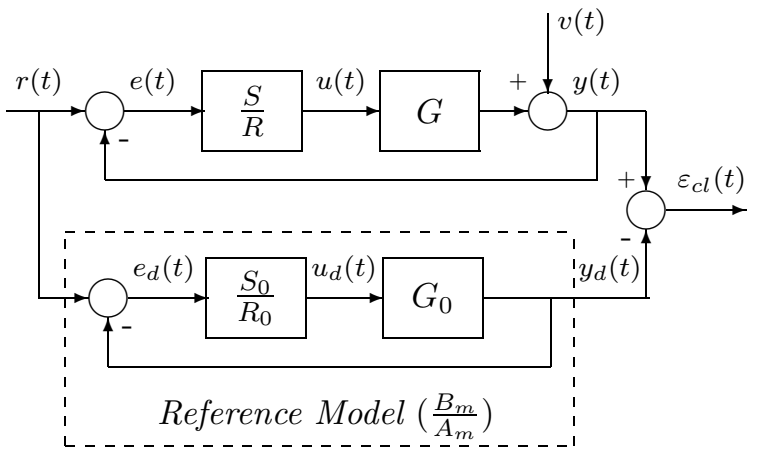

Fig. 1. Block diagram of the achieved and designed closed-loop systems

Figure 1 shows the block diagram of the closedloop system. The upper part represents the achieved closed-loop system and the lower part shows the reference model $\left(B_{m} / A_{m}\right)$ which is presented as the desired closed-loop system containing the initial model of the plant $\left(G_{0}\right)$ and the initial controller $\left(R_{0}, S_{0}\right)$. It is assumed that the initial controller is able to meet the control specifications with respect to the initial model. In this way, the reference model gets a reasonable and attainable structure.

Let the initial controller $\left(R_{0}, S_{0}\right)$ be applied to the real system excited by the reference signal $r(t)$ and the plant output be measured. Then, the closedloop output error (see Fig. 1) defined as

$$
\varepsilon_{c l}(\rho, t)=y(\rho, t)-y_{d}(t)
$$

contains the effect of both modeling errors and noise. Evidently, the effect of modeling errors is correlated with the reference signal, while that of noise is not. Since the lack of control performance results essentially from the modeling errors, an improved controller should be able to compensate the effect of the modeling errors to the point that the closed-loop output error contains only filtered noise. Thus, a reasonable way to tune the controller parameters is to make the closed-loop output error independent of the reference signal. So, the parameters of the controller should be solution to the following $n_{\rho}$ correlation equations:

$$
f(\rho)=\frac{1}{N} \sum_{t=1}^{N} \zeta(\rho, t) \varepsilon_{c l}(\rho, t)=0
$$

where $N$ is the number of data and $\zeta(\rho, t)$ is a $n_{\rho^{-}}$ dimensional vector of instrumental variables. The instrumental variables should be correlated with the reference signal and uncorrelated with noise.

Equation (9) is in general nonlinear and cannot be solved analytically. Iterative numerical solution is possible using the relationship:

$$
\rho_{i+1}=\rho_{i}-\gamma_{i}\left[Q_{N}\left(\rho_{i}\right)\right]^{-1} f\left(\rho_{i}\right)
$$

where $\gamma_{i}$ is the step size and $Q_{N}\left(\rho_{i}\right)$ is a square matrix of dimension $n_{\rho}$. For faster convergence 
one can use the Newton-Raphson method. In this method, $Q_{N}\left(\rho_{i}\right)$ is defined as the derivative of the correlation equation:

$$
\begin{aligned}
Q_{N}\left(\rho_{i}\right)= & \frac{1}{N} \sum_{t=1}^{N}\left\{\left.\frac{\partial \zeta(\rho, t)}{\partial \rho}\right|_{\rho=\rho_{i}} \varepsilon_{c l}\left(\rho_{i}, t\right)+\right. \\
& \left.\left.\zeta\left(\rho_{i}, t\right) \frac{\partial \varepsilon_{c l}(\rho, t)}{\partial \rho}\right|_{\rho=\rho_{i}}\right\}
\end{aligned}
$$

The gradient of the closed-loop output error with respect to $\rho$ can be represented in terms of the regressor vector $\phi$ as follows (Åström and Wittenmark, 1989):

$$
\psi^{T}(\rho, t)=\frac{\partial \varepsilon_{c l}(\rho, t)}{\partial \rho}=\frac{B\left(q^{-1}\right)}{P\left(q^{-1}\right)} \phi^{T}(\rho, t)
$$

where $P\left(q^{-1}\right)=A\left(q^{-1}\right) R\left(q^{-1}\right)+B\left(q^{-1}\right) S\left(q^{-1}\right)$ is the closed-loop characteristic polynomial. Since the plant model is unknown, an estimate $\bar{\psi}$ of this gradient can be used instead (see the definition in Eq. (23)). On the other hand, near the solution, the first term in Eq. (11) is close to zero because the derivatives of the instrumental variables are uncorrelated with the closed-loop output error. Neglecting this term, let redefine $Q_{N}\left(\rho_{i}\right)$ as:

$$
Q_{N}\left(\rho_{i}\right)=\frac{1}{N} \sum_{t=1}^{N} \zeta\left(\rho_{i}, t\right) \bar{\psi}^{T}\left(\rho_{i}, t\right)
$$

Choice of instruments: An "idealized" choice is a noise-free estimate of the gradient $\psi(\rho, t)$ based only on the reference signal (Söderström and Stoica, 1983). This makes $Q_{N}(\rho)$ as close as possible to a positive semi-definite matrix. The instruments can be obtained in two different ways by filtering a noise-free estimate of the regressor:

(1) The first approach is based on identified models, and the corresponding Iterative Correlation-based Tuning will be labeled ICT-IM:

$$
\zeta_{I M}(\rho, t)=\hat{\psi}(\rho, t)=\frac{\hat{B}}{\hat{P}} \hat{\phi}(\rho, t)
$$

where

$$
\begin{aligned}
\hat{\phi}^{T}(\rho, t)= & {\left[-\hat{u}(t-1) \cdots-\hat{u}\left(t-n_{R}\right),\right.} \\
& \left.\hat{e}(t) \cdots \hat{e}\left(t-n_{S}\right)\right]
\end{aligned}
$$

and

$$
\hat{u}(t)=\frac{\hat{A} S}{\hat{P}} r(t) \quad, \quad \hat{e}(t)=\frac{\hat{A} R}{\hat{P}} r(t)
$$

The closed-loop models $\frac{\hat{A} S}{\hat{P}}$ and $\frac{\hat{A} R}{\hat{P}}$ can be identified using open-loop identification methods or they may be computed using the plant model identified in closed loop (Landau and Karimi, 1997) and knowledge of the controller.
(2) The second approach uses the designed output, leading to the acronym ICT-DO:

$$
\zeta_{D O}(\rho, t)=\psi_{d}(\rho, t)=\frac{B_{m}}{A_{m} S} \phi_{d}(\rho, t)
$$

where

$$
\begin{aligned}
\phi_{d}^{T}(\rho, t)= & {\left[-u_{d}(t-1) \cdots-u_{d}\left(t-n_{R}\right),\right.} \\
& \left.e_{d}(t) \cdots e_{d}\left(t-n_{S}\right)\right]
\end{aligned}
$$

and

$$
u_{d}(t)=\frac{S}{R} e_{d}(t) \quad, \quad e_{d}(t)=r(t)-y_{d}(t)
$$

Notice that the instrumental variables $\zeta_{D O}(\rho, t)$ are independent of the noise and the plant model. This approach can be implemented if the controller has no zeros or poles outside the unit circle.

Both choices of instrumental variables can be expressed in the following general form:

$$
\begin{aligned}
\zeta^{T}(t)= & F\left(q^{-1}\right)\left[-\frac{S}{R} r(t-1) \ldots-\frac{S}{R} r\left(t-n_{R}\right),\right. \\
& \left.r(t) \ldots r\left(t-n_{S}\right)\right]
\end{aligned}
$$

where $F\left(q^{-1}\right)$ is an asymptotically stable filter. Therefore, for ICT-IM one has $F=D \frac{\hat{A} R}{\hat{P}}, D=\frac{\hat{B}}{\hat{P}}$ and for ICT-DO $F=D \frac{A_{m}-B_{m}}{A_{m}}, D=\frac{B_{m}}{A_{m} S}$.

\section{CONVERGENCE AND CONSISTENCY}

This section discusses the limiting behavior of the controller parameters $\rho_{i}$ as the number of data tends to infinity. When dealing with consistency, the concept of convergence with probability one (w.p.1) to the true controller parameters is considered. The methods of analysis used here are adopted from the framework used in (Söderström and Stoica, 1981).

Let introduce a number of assumptions about the true system, the controller structure and the experimental conditions under which the data are collected.

(A1) The system to be controlled is SISO, linear time-invariant, finite order and strictly causal.

(A2) The disturbance $v(t)$ is a stationary stochastic process with zero mean and a rational, nonsingular spectral density matrix.

(A3) The reference signal $r(t)$ is persistently exciting of sufficiently high order, and uncorrelated with the disturbance $v(s) \forall s, t$.

(A4) The controller computed at each iteration stabilizes the closed-loop system.

(A5) The order of the estimated controller $\left(n_{R}\right.$ and $\left.n_{S}\right)$ and the order of a controller $\left(n_{R}^{*}\right.$ and $n_{S}^{*}$ ) that is solution to the correlation equation are related by the following inequality:

$$
\min \left(n_{R}-n_{R^{*}}, n_{S}-n_{S^{*}}\right) \geq 0
$$


(A6) The solution $\rho^{*}$ to the correlation equation is unique.

Assumptions A1 and A2 define the class of systems and disturbances to be considered, while A3 is a classical assumption for the excitation signal in parameter estimation algorithms based on the correlation approach. The only additional assumption compared with the classical IV methods for model identification is A4. This assumption may be rather restrictive for some systems, but it is required for implementing the controller on the real system in each iteration. In practice, a stability test based on the initial model of the plant or the model identified in the previous iteration can be performed before implementing a controller. If the stability test fails, the step size $\gamma_{i}$ is reduced so as to obtain a stabilizing controller. The stability test can also be performed without using the plant model, based on the Vinnicombe gap, as it is proposed for the IFT approach in (Kammer et al., 2000).

Assumption A5 implies that there is at least one solution to the correlation equation and this solution is attainable by the estimates. This assumption is required for parameter convergence. However, it is also well known that overparameterization of the controller leads to numerical difficulties due to zero-pole cancellation. Assumptions A6 is necessary only for the consistency analysis and it also implies the equality in (20).

The sufficient conditions for convergence (under Assumptions A1-A5) and consistency (under Assumptions A1-A6) of the iterative parameter update equation (10) are the same as those for conventional parameter estimation methods based on the correlation approach (Ljung, 1987). That is:

$$
Q=\lim _{N \rightarrow \infty} \frac{1}{N} \sum_{t=1}^{N} \zeta(\rho, t) \bar{\psi}^{T}(\rho, t)
$$

exists and is nonsingular w.p.1, and

$$
\lim _{N \rightarrow \infty} \frac{1}{N} \sum_{t=1}^{N} \zeta(\rho, t) v(t)=0 \quad \text { w.p.1. }
$$

where

$$
\bar{\psi}^{T}(\rho, t)=D\left(q^{-1}\right) \phi^{T}(\rho, t)
$$

is an estimation of the gradient vector $\psi$ (defined in 12). After some straightforward calculations, $\bar{\psi}$ can be expressed as:

$$
\bar{\psi}^{T}(\rho, t)=\frac{A D}{P}\left[r(t) \cdots r\left(t-n_{\rho}+1\right)\right] \mathcal{S}^{T}
$$

where $\mathcal{S}$ is defined as:

$$
\mathcal{S}=\left[\begin{array}{cccccc}
0 & -s_{0} & \cdots & -s_{n_{S}} & & \\
& & \ddots & & \ddots & 0 \\
0 & & & -s_{0} & \cdots & -s_{n_{S}} \\
1 & r_{1} & \cdots & r_{n_{R}} & & \\
& \ddots & \ddots & & \ddots & 0 \\
0 & & 1 & r_{1} & \cdots & r_{n_{R}}
\end{array}\right]
$$

Under Assumption A2, the limits in (21) and (22) can be replaced by the corresponding expected values (Söderström and Stoica, 1983):

$$
\begin{aligned}
E \zeta(\rho, t) \bar{\psi}^{T}(\rho, t) & =Q \\
E \zeta(\rho, t) v(t) & =0 .
\end{aligned}
$$

Note that, under Assumption A3, Eq. (26) is trivially satisfied. The conditions of nonsingularity of $Q$ for different types of excitations are given in the following theorem:

Theorem 1. Consider the matrix $Q$ in Eq. (25) and the transfer function $H\left(z^{-1}\right)$ defined as:

$$
H\left(z^{-1}\right)=\frac{F\left(z^{-1}\right)}{R\left(z^{-1}\right) D\left(z^{-1}\right)} \frac{P\left(z^{-1}\right)}{A\left(z^{-1}\right)}
$$

Suppose that Assumptions A1-A5 hold.

(a) If $r(t)$ is persistently exciting of order $\rho$ and $H\left(z^{-1}\right)$ (after zero-pole cancellation) is a strictly positive real transfer function, then the matrix $Q$ is nonsingular.

(b) If $r(t)$ is a deterministic periodic signal with period $\rho$ and persistently exciting of order $\rho$ and $H\left(z^{-1}\right)$ (after zero-pole cancellation) has no pole on the unit circle, then the matrix $Q$ is nonsingular.

The proof of the part (a) of the theorem is based on the following lemma (Söderström and Stoica, 1981):

Lemma 1. Let $\Psi(t)=[x(t-1) \ldots x(t-p)]^{T}$ be a $p$-dimensional stationary stochastic process. Assume that $x(t)$ is persistently exciting of order $p$. Let the scalar filter $H\left(z^{-1}\right)$ be a strictly positive real (SPR) transfer function. Then the matrix $Z=E\left[H\left(z^{-1}\right) \Psi(t)\right] \Psi^{T}(t)$ is nonsingular.

Proof of Theorem 1: Taking into account the relation (24), the general form of $Q$ is:

$$
Q=E \frac{F\left(q^{-1}\right)}{R\left(q^{-1}\right)}\left[\begin{array}{c}
-S\left(q^{-1}\right) r(t-1) \\
\vdots \\
-S\left(q^{-1}\right) r\left(t-n_{R}\right) \\
R\left(q^{-1}\right) r(t) \\
\vdots \\
R\left(q^{-1}\right) r\left(t-n_{S}\right)
\end{array}\right]
$$

$$
\times\left[r_{f}(t) \cdots r_{f}\left(t-n_{\rho}+1\right)\right] \mathcal{S}^{T}
$$


where

$$
r_{f}(t)=\frac{A D}{P} r(t)
$$

This matrix can also be presented as:

$$
Q=\mathcal{S} \cdot \mathcal{T} \cdot \mathcal{S}^{T}
$$

where the matrix $\mathcal{T}$ is defined by:

$$
\begin{aligned}
\mathcal{T}= & E \frac{F\left(q^{-1}\right)}{R\left(q^{-1}\right)}\left[r(t) \cdots r\left(t-n_{\rho}+1\right)\right]^{T} \\
& \times\left[r_{f}(t) \cdots r_{f}\left(t-n_{\rho}+1\right)\right]
\end{aligned}
$$

It results from Eq. (29) that $Q$ is nonsingular if and only if the matrices $\mathcal{T}$ and $\mathcal{S}$ are nonsingular. As for the $\mathcal{S}$ matrix, it is well known in the theory of resultants (van der Waerden, 1991) that $\mathcal{S}$ is nonsingular if and only if the polynomials $R$ and $S$ are coprime (this condition will be satisfied under Assumption A5 with the equality in 20). Thus, $Q$ is nonsingular if and only if $\mathcal{T}$ is nonsingular. But $\mathcal{T}$ can be expressed as:

$$
\begin{aligned}
\mathcal{T}= & E \frac{F\left(z^{-1}\right)}{R\left(z^{-1}\right) D\left(z^{-1}\right)} \frac{P\left(z^{-1}\right)}{A\left(z^{-1}\right)}\left[\begin{array}{c}
r_{f}(t) \\
\vdots \\
r_{f}\left(t-n_{\rho}+1\right)
\end{array}\right] \\
& \times\left[r_{f}(t) \cdots r_{f}\left(t-n_{\rho}+1\right)\right]
\end{aligned}
$$

Now Lemma 1 can be applied to show that $\mathcal{T}$ is nonsingular if $H\left(z^{-1}\right)$ is SPR. Note that, under this condition, $r_{f}(t)$ is also persistently exciting of order $\rho$ because $H\left(z^{-1}\right)$ has no zeros on the unit circle.

The proof of part (b) of the Theorem goes along the lines of the proof of Theorem 5.1, part (iii) in (Söderström and Stoica, 1981) and will not be given here.

\section{Remarks:}

(1) The transfer function $H\left(z^{-1}\right)$ for ICT-IM variant becomes:

$$
H\left(z^{-1}\right)=\frac{\hat{A}\left(z^{-1}\right)}{A\left(z^{-1}\right)} \frac{P\left(z^{-1}\right)}{\hat{P}\left(z^{-1}\right)}
$$

It is clear that when $\hat{A}=A$ and $\hat{P}=P$, this transfer function is SPR. However, with a good estimation of the closed-loop system, the strictly positive realness of $H$ is strongly expected. Yet, it is interesting to mention that poor estimates of $A$ and $P$ might as well give a consistent algorithm if the SPR condition is satisfied. In this case, only the convergence speed is affected because a good estimation of the filter $\hat{B} / \hat{P}$ preserves the gradient descent direction and improves the speed of convergence. This will be illustrated by a simulation example in Section 4 .

(2) For ICT-DO variant, one has:

$$
H\left(z^{-1}\right)=\frac{P\left(z^{-1}\right)}{A\left(z^{-1}\right) R\left(z^{-1}\right)} \frac{A_{m}\left(z^{-1}\right)-B_{m}\left(z^{-1}\right)}{A_{m}\left(z^{-1}\right)}
$$

It can be observed that this transfer function is independent of the identified plant model. On the other hand, in the proximity of the optimal solution, where $A_{m} \approx P$ and $A_{m}-$ $B_{m} \approx A R$, the transfer function $H$ is likely SPR. Therefore, this variant seems to be suitable for systems with large unmodeled dynamics and noise in final iterations.

(3) Part (b) of Theorem 1 shows that with a periodic signal of period $\rho$ as the excitation signal, the method will be consistent for all $A, B, P$ and their estimates with a much weaker condition on $H$. However, if for practical reasons this type of signal is not implementable on the real system, Part $(a)$ that is valid for all persistently exciting $r(t)$ of at least order $\rho$ may be used.

It should be mentioned that, in practice when the number of data $N$ is finite, the solution to the correlation equation changes in each iteration because of different noise realization (this change tends to zero when $N$ tends to infinity). However, when the number of iterations goes to infinity, the expectation of the estimates tends to the true values (the solution with infinite number of data). As a result, the proposed iterative controller tuning method needs more iterations for convergence compared with the IV methods for model parameter estimation where only one data collection is used in all iterations.

\section{SIMULATION RESULTS}

The aim of this section is to provide two simulation examples in order to illustrate the theoretical results of Section 3. In the first simulation the influence of modeling errors on the convergence speed in the absence of noise is investigated. The second simulation compares the behavior of ICTIM and ICD-DO variants in the presence of noise via Monte-Carlo simulation.

The following system is considered:

$$
\begin{gathered}
\left(1-1.5 q^{-1}+0.7 q^{-2}\right) y(t)=\left(q^{-1}+0.5 q^{-2}\right) u(t) \\
+\left(1+0.5 q^{-1}+0.5 q^{-2}\right) e(t)
\end{gathered}
$$

where $e(t)$ is zero-mean, stationary, white Gaussian noise with variance $\lambda^{2}$ (for the first simulation $\lambda=0)$. The reference model is given by:

$$
\frac{B_{m}}{A_{m}}=\frac{-0.0781 q^{-1}-0.0625 q^{-2}-0.0117 q^{-3}}{1-1.5781 q^{-1}+0.6375 q^{-2}-0.0117 q^{-3}}
$$


which has two poles at 0.7794 and one pole at 0.019. Using the pole-placement technique, the optimal controller can be easily computed as: $R^{*}\left(q^{-1}\right)=1$ and $S^{*}\left(q^{-1}\right)=-0.0781-0.0234 q^{-1}$ which gives $\rho^{*}=\left[\begin{array}{ll}-0.0781 & -0.0234\end{array}\right]^{T}$. The same structure is considered for the initial controller with the initial parameter vector $\rho_{0}=\left[\begin{array}{ll}0.075 & 0\end{array}\right]^{T}$ which represents a proportional controller that stabilizes the closed-loop system.

Consider first the ICT-IM variant where the closed-loop models used for filtering $\left(\frac{\hat{A} S}{\hat{P}}, \frac{\hat{A} R}{\hat{P}}, \frac{\hat{B}}{\hat{P}}\right)$ in (14) and (16) are computed using the current controller and the plant model $\left(\frac{\hat{B}}{\hat{A}}\right)$ identified in closed loop. The reference signal $r(t)$ is a PRBS generated by an 11-bit shift register (data length $\mathrm{N}=2047$ ). Table 1 gives the number of iterations needed to achieve a parametric distance of $1 \mathrm{e}-9$, defined as $P D=\left(\rho_{i}-\rho^{*}\right)^{T}\left(\rho_{i}-\rho^{*}\right)$, for different orders of the polynomials $\hat{A}$ and $\hat{B}$.

Table 1. Influence of the modeling error

\begin{tabular}{|c|c|c|c|c|c|}
\hline \hline$n_{\hat{A}}=\operatorname{deg}(\hat{A})$ & 0 & 1 & 1 & 2 & 2 \\
$n_{\hat{B}}=\operatorname{deg}(\hat{B})$ & 1 & 1 & 2 & 1 & 2 \\
\hline No. iter. & 55 & 11 & 9 & 6 & 5 \\
\hline \hline
\end{tabular}

It is clearly seen that the speed of convergence depends on the order of the identified plant model. Note, however, that ICT-IM variant gives consistent estimates even in the case when the plant is modeled only by a gain $\left(n_{\hat{A}}=0\right.$ and $\left.n_{\hat{B}}=1\right)$.

The second simulation study illustrates the behavior of the ICT-IM and ICD-DO variants in the presence of noise. To compare ICT-IM and ICT-DO variants 100 Monte-Carlo simulations are performed. For each simulation run, 20 iterations are carried out and each iteration is performed with a different realization of the noise $e(t)$ that provides a ratio noise/signal of about $7,5 \%$ in terms of variance. The same PRBS as in the previous numerical example is used as the reference signal. The plant model for the ICT-IM variant is identified with $n_{\hat{A}}=1$ and $n_{\hat{B}}=1$. For the first 10 iterations, the ICT-IM variant is used and in the next 10 iterations, when the estimates are close to the solution, the two variants are compared.

Let define the parametric error as $\Delta \rho_{j}=\rho_{j}^{*}-$ $\rho_{j} ; j=0,1$. Table 2 shows the mean values and variances of the parametric errors over 100 simulation runs for both the ICT-IM and ICT-DO variants.

It can be seen that both variants provide the convergence to the optimal values in the presence

Table 2. Comparison of IV variants

\begin{tabular}{|c|c|c|c|c|}
\hline \hline & \multicolumn{2}{|c|}{ ICT-IM } & \multicolumn{2}{c|}{ ICT-DO } \\
\cline { 2 - 5 } & $\operatorname{mean}\left(\Delta \rho_{j}\right)$ & $\operatorname{var}\left(\Delta \rho_{j}\right)$ & $\operatorname{mean}\left(\Delta \rho_{j}\right)$ & $\operatorname{var}\left(\Delta \rho_{j}\right)$ \\
\cline { 2 - 5 }$\rho_{0}$ & $-2.71 \mathrm{e}-3$ & $5.96 \mathrm{e}-5$ & $-6.81 \mathrm{e}-4$ & $2.11 \mathrm{e}-5$ \\
$\rho_{1}$ & $2.97 \mathrm{e}-3$ & $6.64 \mathrm{e}-5$ & $7.35 \mathrm{e}-4$ & $2.15 \mathrm{e}-5$ \\
\hline \hline
\end{tabular}

of noise. Note also that, in the proximity of the solution, ICT-DO variant is less sensitive to noise and shows better performance in terms of meanvalue and variance of the parametric error. This suggests using the ICT-IM variant in few first iterations and then switching to the ICT-DO variant.

\section{CONCLUSIONS}

It has been shown that making the output error between the closed-loop system and a reference model uncorrelated with reference signal, can be used as objective for controller tuning in modelfollowing problems. The iterative correlationbased tuning (ICT) approach preserves the designed objectives, presented in terms of a reference model, independently of the noise characteristics. The algorithm requires an approximate model of the plant for computing the gradient of the output error. However, the convergence analysis shows that modeling errors do not affect the parametric convergence as long as a SPR condition on some transfer function is satisfied. Simulation examples illustrate well the theoretical results regarding the consistency of the proposed method.

\section{REFERENCES}

Åström, K. J. and B. Wittenmark (1989). Adaptive Control. Addison-Wesley.

Hjalmarsson, H., S. Gunnarsson and M. Gevers (1994). A convergent iterative restricted complexity control design scheme. In: 33rd IEEE$C D C$. Vol. 2. pp. 1735-1740.

Kammer, L. C., R. R. Bitmead and P. L. Bartlett (2000). Direct iterative tuning via spectral analysis. Automatica 36(9), 1301-1307.

Landau, I. D. and A. Karimi (1997). Recursive algorithms for identification in closed loop - a unified approach and evaluation. Automatica 33(8), 1499-1523.

Ljung, L. (1987). System Identification - Theory for the User. Prentice Hall. NJ, USA.

Söderström, T. and P. Stoica (1981). Comparison of some instrumental variable methods consistency and accuracy aspects. Automatica 17(1), 101-115.

Söderström, T. and P. Stoica (1983). Instrumental variable methods for system identification. In: Lecture Notes in Control and Information Science (A. V. Balakrishnan and M. Thoma, Eds.). Springer-Verlag. Berlin.

Trulsson, E. and L. Ljung (1985). Adaptive control based on explicit criterion minimization. Automatica 21(4), 385-399.

van der Waerden, B. L. (1991). Algebra. vii ed.. Springer-Verlag. New York. 